\title{
Differential weighting and directed meaning as explanations of primacy in impression formation ${ }^{1,2}$
}

\author{
ABRAHAM TESSER, DEPARTMENT OF PSYCHOLOGY, \\ UNIVERSITY OF GEORGIA, Athens, Ga. 30601
}

An experiment designed to test a Gestaltist shift in meaning explanation and a differential weighting explanation of primacy was performed. Primacy had a significant $(p<.05)$ effect on overall impression but there was no evidence of shift in meaning of traits as a function of position in the list. Only partial support was found for the differential weighting hypothesis.

A number of investigators have demonstrated a primacy effect in personality impression formation tasks. They have found that Ss are more influenced by early information than later information when forming impressions of stimulus persons. The dynamics of this effect, however, are not clearly understood.

- Two explanations have been offered. One, which may be termed the "directed impression" hypothesis, has been advanced by Asch (1946). He argues that when Ss form an impression from a list of adjectives"...the first terms set up in most subjects a direction which then exerts a continuous effect on the latter terms [pp. 271-272]." That is, the meaning of the latter words are shaded so as to be consistent with the first words.

A differential weighting explanation of the primacy phenomenon has been offered by Anderson (e.g., Anderson \& Hubert, 1963). He argues that it is not the meaning of the latter adjectives that changes, it is their relative weights in the overall impression.

The purpose of this study is to provide a direct test of the directed impression hypothesis and to allow an evaluation of the differential weighting hypothesis. Meaning was operationally defined as the score obtained across four semantic differential bipolar scales which are highly loaded on an evaluative factor (Osgood, Suci, \& Tannenbaum, 1957).

A test of the directed impression explanation would consist of examining the meaning of a word as it occupies different positions in a given bivalent list. If this explanation holds, we would expect a positively evaluated adjective to maintain its positiveness at the beginning of a list and to lose some of its positivity if it is preceded by negatively evaluated adjectives. Similarly, a negatively evaluated adjective should maintain its negativeness in the beginning of the list and should become less negative when preceded by positively evaluated adjectives.

In addition, $\mathrm{S}$ should make equal use of a given word (after its meaning has been shaded) in forming his total impression regardless of its position in the list. One index of the extent of usage of an adjective in forming the overall impression is the correlation between the adjective and the total impression. The prediction under the directed meaning hypothesis, then would be that the correlation between the evaluative aspect of a given adjective in a given list and the overall evaluative flavor of the impression formed from that list should remain constant regardless of the serial position of the adjective.

On the other hand, the differential weighting explanation predicts that the meanings should remain constant regardless of serial position. The Ss should, however, in forming their overall impressions use the first words in the list more than the last words. Thus, under this hypothesis we would expect that the correlation between a given adjective in a given list and the overall impression formed from that list would decrease as the adjective moves from the beginning of the list to the end of it.

Method. One hundred and twenty-nine $\mathrm{Ss}^{3}$ served in this study. They were all male, undergraduate, college students from a large midwestern university. Eighty adjectives were scaled by all the Ss on four, seven-step, evaluative semantic differential scales (Good-Bad, Nice-Awful, Beautiful-Ugly, FairUnfair) using semantic differential instructions under the set to "Think of the meaning of each adjective as it would be used to describe a person ....."

On the basis of this scaling eight Ps (positively evaluated traits) and eight
Ns (negatively evaluated traits) were selected to yield maximum divergence between the Ps and the Ns.

Three words were randomly chosen from both the eight Ps and eight Ns yielding three $P_{s}$ and three Ns to be used in the impression formation list [Considerate $\left(\mathrm{P}_{1}\right)$, Truthful $\left(\mathrm{P}_{2}\right)$, Understanding $\left(\mathrm{P}_{3}\right)$, Mean $\left(\mathrm{N}_{1}\right)$, Spiteful $\left(\mathrm{N}_{2}\right)$, Underhanded $\left.\left(\mathrm{N}_{3}\right)\right]$, and the 10 remaining words served as a check on the reliability of the experimental words.

The six experimental words were put into six orders thereby generating six separate lists. The criteria used for generating the orders are as follows: (1) The three Ps and three Ns must always appear in homogeneous blocks; (2) For half the lists, a block of three Ps must precede a block of three Ns and vice versa for the other half; (3) Each word must appear in each position within the lists, i.e., $P_{1}$, for example, must appear in Position 1, Position 2, etc.

The purpose for including criteria (1) and (2) was to provide the necessary conditions to assess a primacy effect. Criterion 3 was included so that systematic positional effects on word meanings or weighings could be assessed.

Each list order was used as the stimulus adjective list for the impression formation task for one group of Ss. Thus, there was a total of six groups, each getting a different list order. The groups were formed by randomly dividing $126^{3}$ Ss who scaled the preliminary 80 adjectives into six groups. Ss completed the final part of the study from two to four weeks after they completed the preliminary scaling.

The final and major part of the study can be broken down into three parts. First Ss responded to each of the reliability words on the four semantic differential scales. Next they were read a list of six neutral words ${ }^{4}$, and they were asked to form an impression of the person described by these words. They recorded their impression on the set of four semantic differential scales. The list was then repeated. This time they responded after each word with their impression of the meaning of that word as it described that particular individual. The purpose of including the neutral list was to give the Ss practice in and to have them familiarize themselves with the procedure to be used with the experimental list. The experiment was completed with a repetition of the tasks performed above using the experimental list instead of the neutral list. The neutral list was the same for all six groups. The order of the experimental list was varied for each group.

Results and Discussion. A test-retest reliability coefficient (Pearson product-moment) was computed for each reliability word and the reliability seemed adequate $(\mathrm{r}=.52)$. Primacy was found significant at the .05 level $(F=4.32$, df $=1 / 120)$.

If the directed meaning explanation is valid, one should find that as each of the words move from the first half of the list to the second half there is a difference in evaluative meaning as assessed by the semantic differential. In order to test this prediction six single factor, six level analyses of variance were computed. Each analysis was based on a single word. The positions in the list constituted the six levels for each analysis. The directed impression hypothesis failed to receive support. The word meaning scores for each word do not differ as a function of position in the list as predicted.

These data do provide some weak support for the differential weighting hypothesis. This hypothesis predicts no difference among means as a function of list position, i.e., acceptance of the null hypothesis. This, however, is indirect support, depending in this way upon the null hypothesis. If the differential weighting explanation holds, we should find that a word appearing in the first half of the list correlates more highly with the overall impression than when that same word appears in the second half of the list.

In order to gain power in testing the above predictions the groups that had the Ps in the first half of the list were combined. The groups that had the Ns in the first half of the list were also combined. For each combined group, a product-moment correlation was computed between the word meaning score after it had appeared in the list and the overall impression. 
Let us first examine the three Ps. When each of these words appear in the first half of the list, the correlations of their meaning scores with the overall impression are significant $(r=.32, .21, .22)$ while these correlations are essentially zero $(r=.00, .01,-.01)$ when the words are in the last half of the list. This result is consistent with the differential weighting hypothesis which also predicts a difference between the two correlations for each word. Confirmation of this prediction was consistent though the significances of the differences were not very high $(\mathrm{P}=.03, .13$, $.10)$.

Looking now at the Ns, none of the correlations differ significantly from zero. This result is not consistent with either hypothesis.

In conclusion no evidence was found for the directed impression hypothesis, and only very limited evidence for the differential weighting hypothesis was found. This is not to say that both are completely wrong. It does seem to indicate, however, that these hypotheses without some revision or qualification are inadequate as explanations of the primacy effect in impression formation.

\section{REFERENCES}

ANDERSON, N. H., \& HUBERT, S. Effects of concomitant verbal recall on order effects in personality impression formation. J. verbal Learn. verbal Behav., 1963, 2, 379-391.

ASCH, S. E. Forming impressions of personality. J. abnorm soc. Psychol, 1946, 41, 258-290.

OSGOOD, C. E., \& TANNENBAUM, P. H. The principle of congruity in the prediction of attitude change. Psychol. Rev., 1955, 62, 42-45.

TESSER, A. An investigation of the primacy effect in impression formation. Unpublished master's thesis, Purdue University, 1965.

\section{NOTES}

1. This paper is based on a Master's Thesis submitted to the Graduate School of Purdue University in partial fulfillment of the requirements for the Master of Science degree. The author is grateful to Karl Weick who supervised the research and contributed many helpful suggestions.

2. An earlier version of this paper was presented at the fortieth annual meeting of the Midwestern Psychological Association, Chicago, May 4, 1968.

2 . Three out of the original 129 Ss failed to complete the experiment.

4. These neutral words were selected from a list of 555 adjectives scaled for likeableness (Anderson, 1964). The words used, in the order in which they appeared in the list, along with their scaled scores are: Impulsive (3.07), Hesitant (2.90), Quiet (3.11), Shy (2.91), Changeable (2.97), Aggressive (3.04). A score of 6.00 was the most favorable rating possible, .00 was the least favorable rating possible. 